\title{
Design, materiais e sustentabilidade: micronização de produto multi-material visando sua reciclagem
}

\section{Design, materials and sustainability: multi-material product micronization for recycling}

\author{
Elisa Guerra Ashton ${ }^{1}$ \\ Wilson Kindlein Junior ${ }^{2}$ \\ Luis Henrique Cândido ${ }^{3}$ \\ Renan Demori ${ }^{4}$ \\ Luiz Carlos Robinson ${ }^{5}$ \\ Raquel Mauler
}

ISSN: 1808-3129 


\section{Resumo}

A aplicação do ecodesign na seleção de materais é de extrema importância. Entretanto, percebe-se uma tendência contrária a essa prática, a de utilização de diversos materiais em um componente do produto (chamado multi-materiail). Apesar de apresentarem vantagens de fabricação,a reciclagem dos multi-materiais é complexa devido à dificuldade de separação dos materiais. Nesse contexto, apresenta-se como hipótese a utilização da micronização como alternativa para viabilizar o reprocessamento dos multi-materiais. Assim sendo, essa pesquisa buscou analisar a possibilidade de aplicação da micronização na reciclagem de produtos multi-materias co-injetados. Para tanto, o estudo prático consistiu na micronização de escovas de dente multi-materiais e posterior extrusão com polietileno de baixa densidade linear (PEDBL) virgem. Os resultados demonstraram que a micronização pode ser utilizada na reciclagem desses produtos, uma vez que o processo analisado se mostrou factível, utilizando métodos acessíveis. Além disso, não houve degradação do material ao longo do processo, assim como, o mesmo não danificou os equipamentos.

Palavras-Chave: Multi-materiais; Reciclagem; Sustentabilidade; Design.

\footnotetext{
1 Universidade Federal do Rio Grande do Sul - UFRGS RS - Brasil

elisaashton@hotmail.com

${ }^{2}$ Universidade Federal do Rio Grande do Sul - UFRGS RS - Brasil

wilsonkindleinjunior@gmail.com

${ }^{3}$ Universidade Federal do Rio Grande do Sul - UFRGS

RS - Brasil

candido@ufrgs.br

${ }^{4}$ Universidade Federal do Rio Grande do Sul - UFRGS

RS - Brasil
}

\section{Abstract}

Ecodesign application in material selection is very important. However, there is a contrary trend: the combined use of different materials in one component (called multi-materials). Despite these multi-material products present several manufacturing advantages, its recycling is compromised due to the difficulty in separating the product materials. In this context, it is presented as a hypothesis the use of micronization as a possible alternative for processing multi-material products. Hence, this research aimed to analyze the possibility of applying micronization in co-injected multi-materials toothbrushes recycling. Therefore, the practical study consisted in micronization and subsequent extrusion of the multi-material tooth brushes with virgin linear low density polyethylene (LLDPE). The results demonstrated that micronization can be used for recycling multi-material toothbrushes, once the analyzed process proved to be viable, using affordable methods. Furthermore, there was no material degradation during the process, additionally the material did not damage the equipment used.

Keywords: Multi-materials; Recycling; Sustainability; Product Design.

re.demori@hotmail.com

${ }^{5}$ Universidade Feevale

Brasil

luiz.robinson@feevale.br

${ }^{6}$ Universidade Federal do Rio Grande do Sul - UFRGS

RS - Brasil

raquel.mauler@ufrgs.br

Artigo indicado através da organização do evento IDEMi 2015 


\section{INTRODUÇÃO}

A preocupação com as questões ambientais toma proporções cada vez maiores. O aumento nos padrões de consumo da sociedade multiplicado pelo aumento populacional global desencadeia previsões pessimistas em relação à sustentabilidade do planeta (ASHBY; JOHNSON, 2002; ASSADOURIAN, 2010; ONU, 2013; CETELEM, 2011).

Assim sendo, ao considerar o design no contexto da sustentabilidade, grande parte dos autores (WIMMER et al., 2010; MANZINI; VEZZOLI, 2002; MEYER, 2014; HALLSTEDT et al., 2013; GAZIULUSOY et al., 2013; SIMÕES et al., 2013) defende que o design de produto e a seleção de materiais devem ser orientadas conforme preceitos do ecodesign, levando em conta a orientação para a desmontagem (DfD), separação dos materiais e reciclagem (DfR), com o objetivo de reduzir ao máximo o impacto ambiental em todas as fases do ciclo de vida do produto.

Apesar do conhecimento e da necessidade urgente de aplicação do ecodesign no desenvolvimento de produtos, percebe-se que existe uma tendência contrária a essa prática, caracterizada pelo desenvolvimento de produtos multi-materiais. Os produtos com mais de um material em um mesmo componente - chamados de multi-materiais (KROMM et al., 2007), passaram a ser desenvolvidos em decorrência do aumento de requisitos nos produtos e da grande diversidade de materiais existentes (THOMAS; YANG, 2009; WARGNIER et al., 2014).

Um dos principais processos de fabricação dos produtos poliméricos multi-materiais é a moldagem por co-injeção. Esse processo consiste na moldagem simultânea ou sequencial de mais de um tipo de polímero em um mesmo molde durante um único ciclo (ZAVERL et al., 2013). Apesar de a moldagem por co-injeção multi-materiais apresentar diversas vantagens técnicas, como propriedades únicas, facilidade de fabricação e montagem, agilidade no processo etc. (ADVANI; HSIAO, 2012), a mesma dificulta a reciclagem dos produtos, uma vez que a separação dos materiais se torna difícil, e muitas vezes, ineficiente (WORRELL; REUTER, 2014; ALLWOOD; CULLEN, 2012).

Diante do exposto, percebe-se que a situação ideal para a minimização dos impactos ambientais causados pelos produtos, a qual se caracteriza pela ampla aplicação do ecodesign no desenvolvimento dos mesmos, é passível de ser alcançada a longo prazo. Entretanto, existe a necessidade de se buscar também soluções a médio e curto prazo para a minimização dos impactos causados pelos produtos multi-materiais co-injetados. É nesse contexto que o presente estudo se insere.

Assim sendo, apresenta-se como uma possivel solução a curto prazo a reciclagem mecânica de produtos multi-materiais através do processo de micronização. Dessa forma, essa pesquisa buscou analisar a possibilidade de aplicação da micronização na reciclagem de escovas de dente multi-materias co-injetadas.

Adicionalmente, o presente estudo se justifica por apresentar uma contribuição na busca pela sustentabilidade através do desenvolvimento de soluções a curto e médio prazo para a redução dos impactos ambientais causados por produtos multi-materiais. 


\section{DESIGN E SELEÇÃO DE MATERIAIS: CONTEXTO DOS PRODUTOS POLIMÉRICOS MULTI-MATERIAIS}

O design de produto está intimamente ligado à seleção de materiais, sendo essa uma importante etapa no desenvolvimento de produtos (FERRANTE; WALTER, 2010; KARANA et al., 2014; ASHBY; JOHNSON, 2002; MALEQUE; SALIT, 2013). Os materiais, por sua vez, constituem elementos de grande importância para a evolução da sociedade. Confirmando tal colocação, Ashby (2011) destaca os períodos clássicos da evolução da humanidade, cujos nomes são determinados de acordo com o material mais utilizado pela sociedade em cada época: Idade da Pedra, Idade do Cobre, Idade do Ferro etc.

Considerando o panorama evolutivo que relaciona os materiais com a sociedade, Van Bezooyen (2014) defende que a relação do designer com a seleção de materiais também evoluiu dramaticamente. Essa evolução decorreu, principalmente, do surgimento de novos processos e materiais que permitiram uma grande liberdade criativa, como os polímeros, por exemplo. Assim sendo, pode-se considerar que, atualmente, vive-se a Idade dos Polímeros (THOMAS; YANG, 2009).

A partir da Segunda Guerra Mundial, intensificou-se a substituição de materiais como madeira, metais e vidro por polímeros, tendência que persiste até hoje (ALLWOOD; CULLEN, 2012). A crescente preferência na utilização de polímeros em substituição aos materiais tradicionalmente utilizados se deu por diversos motivos, dentre os quais se destacam baixo custo, versatilidade e leveza (JULIER, 2013).

A grande demanda por esses materiais estimulou o surgimento de pesquisas sobre novos tipos de polímeros, novas formas de processamento, novas aplicações etc. Nesse contexto, Thomas e Yang (2009) citam como consequência a grande diversidade de polímeros disponíveis atualmente, que por sua vez, resulta na tendência de utilização conjunta de mais de um tipo de material no mesmo componente.

Os produtos que surgem dessa tendência são os multi-materiais. Tais produtos são definidos como aqueles que possuem uma associação de materiais dispostos de uma maneira previamente definida, ou seja, mais de um material em um mesmo componente (KROMM et al., 2007; WARGNIER et al., 2014). Como exemplo, pode-se citar os produtos desenvolvidos em múltiplas camadas de diferentes materiais, compósitos, co-injetados etc. As principais razões para a utilização de multi-materiais, segundo Wargnier et al. (2014) consistem em: melhorar a performance de um componente, integrar mais funções, reduzir os custos operacionais e facilitar a produção, além de evitar partes muito volumosas.

Consideradas estas vantagens, a utilização de multi-materiais se tornou comum atualmente, com aplicação nos mais diversos produtos, a exemplo de: escova de dente, óculos de sol, lâminas de barbear, painéis automotivos, embalagens de alimentos, partes de aeronaves etc. (WARGNIER et al., 2014). O processo de fabricação tradicionalmente utilizado para os produtos multi-materais consiste na moldagem por co-injeção, também chamada de injeção sanduíche.

De acordo com Zaverl et al. (2013), a moldagem por co-injeção é um processo no qual dois polímeros são injetados simultaneamente ou sequencialmente em um 
mesmo molde. O resultado final desse processo é um produto heterogêneo, composto por um "core material", que seria o material principal, e um "skin material" (material da superfície, aplicado em menor quantidade) que se fundem.

Kim e Isayev (2015) descrevem que a moldagem por co-injeção é conhecida desde o início dos anos 70, quando o processo foi desenvolvido. Segundo os autores, a possibilidade de combinar dois polímeros diferentes pode proporcionar propriedades únicas, que não seriam alcançadas através do processo tradicional com uma única injeção.

Para citar mais algumas vantagens da moldagem por co-injeção de polímeros, destacam-se: a melhora na qualidade do produto final, a redução dos custos e do tempo de produção (ADVANI; HSIAO, 2012), e uma única etapa na fabricação (apenas uma injetora e um molde), características essas que preenchem os requisitos do Design for Manufacture and Assembly (DfMA, ou Design orientado para a fabricação e montage) (BOOTHROYD et al., 2011). O DfMA consiste em um conjunto de métodos voltados para tornar mais rápido e eficiente o processo de fabricação e montagem, além de reduzir custos (PLATCHEK, 2012). A sua aplicação passa a ser imprescindível quando se trata de competitividade e qualidade do produto final (ReVELLE, 2002), sendo esse último mais um motivo pelo qual os multi-materiais co-injetados são vastamente utilizados.

Com uma abordagem voltada para área da engenharia, os autores Kim e Isayev (2015) afirmam que o processo de co-injeção é um dos mais promissores, tanto do ponto de vista econômico, quanto ambiental, já que o material principal pode ser um polímero barato ou reciclado. No que diz respeito à vantagem ambiental, pode-se considerar essa afirmação de Kim e Isayev controversa, se analisada pelo olhar do ecodesign, uma vez que a fusão de dois materiais distintos dificulta sua separação e, portanto, sua reciclagem por meio de processos tradicionais (WORRELL; REUTER, 2014; ALLWOOD, CULLEN, 2012).

\section{RECICLAGEM DE POLÍMEROS E MICRONIZAÇÃO}

Em contraponto ao cenário de crescente utilização de multi-materiais, grande parte da comunidade científica (NKWACHUKWU et al., 2013; RAJENDRAN, 2012; HOPEWELL et al., 2009; KOUSHAL et al., 2014; WORRELL; REUTER, 2014) considera a reciclagem como a melhor opção no gerenciamento dos resíduos poliméricos. Pinto (2012), na página 16, conceitua reciclagem como "todos os possíveis usos de um material, uma vez terminada a vida útil da aplicação pretendida para a peça ou material considerado".

Cabe destacar ainda as diversas vantagens da reciclagem que, de acordo com Rajendran et al. (2012), consistem na redução do consumo de energia e na diminuição da emissão de gás carbônico, já que a reciclagem evita o processo de extração de petróleo e de transformação do polímero (sendo a segunda uma etapa que consome em média $95 \%$ da energia total do processo). Além disso, a reciclagem promove a redução dos volumes enviados a aterro sanitário. Também Hopewell et al. (2009) associa a reciclagem à redução na extração e no consumo de petróleo. 
Apesar da eminente necessidade de expansão das práticas de reciclagem, muitos produtos ainda são desenvolvidos em desacordo com os conceitos de ecodesign ou planejamentos de DfD, o que dificulta o reprocessamento. É o caso dos multi-materiais apresentados no item anterior, cuja reciclagem mecânica é dificultada devido à complexidade de separação dos materiais.

Essa situação acarreta o acúmulo de resíduos sólidos em aterros sanitários. Não obstante, esse tipo de disposição apresenta algumas questões que merecem uma análise crítica. Inicialmente, à medida que o volume de resíduos sólidos gerados aumenta, diminui a capacidade dos aterros. Esses depósitos necessitam de uma extensa área física, o que limita a construção de novos aterros (KOLLIKKATHARA et al., 2010). Da mesma maneira, a inexistência de gerenciamento acaba levando a um tipo de descarte ainda mais prejudicial: o descarte ilegal em locais inadequados como rios, ruas, áreas verdes etc. (ICHINOSE; YAMAMOTO, 2011). Assim sendo, a impossibilidade de reprocessamento de materiais poliméricos culmina no acúmulo de resíduos sólidos, essa situação traz consequências que atingem esferas ambientais (AGUADO; SERRANO, 1999), sociais (WILSON et al., 2006) e econômicas (JUCÁ, 2003).

Diante desse contexto, considera-se que, apesar do aumento da reciclagem de polímeros e do surgimento de novas alternativas para o fim de vida dos mesmos, importantes esforços ainda são necessários para se alcançar a eficiência na reciclagem (JIMÉNEZ et al., 2015). Isso se deve às limitações dos processos existentes, em especial à necessidade de separação e identificação dos materiais para a reciclagem mecânica (ANTUNES, 2007), ao alto custo da reciclagem química (MORSELLI et al., 2009) e à necessidade de extração de agentes tóxicos na recuperação energética (JIMÉNEZ et al., 2015).

Assim sendo, essa pesquisa se propôs a analisar a possibilidade de reciclagem de produtos multi-materiais através da aplicação do processo de micronização, o que dispensa a etapa de desmontagem e separação dos materiais. A micronização é um processo utilizado para redução do tamanho das partículas de um material. Ela pode ser realizada à temperatura ambiente ou por criogenia (LAGARINHOS; TENÓRIO, 2008).

Nessa pesquisa, foi aplicada a micronização à temperatura ambiente. Esse processo é realizado em equipamentos mais robustos do que a criogênica e opera em temperaturas que chegam a $120^{\circ} \mathrm{C}$, por isso não é indicado para materiais com baixo ponto de fusão (LAGARINHOS; TENÓRIO, 2008). De acordo com Ayyer, et al. (2013), a micronização à temperatura ambiente tem capacidade de gerar partículas de aproximadamente $0,4 \mathrm{~mm}$ ou 40 mesh, com poucas partículas mais finas. Já Lagarinhos e Tenório (2008) descrevem que, nesse tipo de micronização, o tamanho final das partículas seria de até $0,21 \mathrm{~mm}$, o equivalente a 70 mesh, aproximadamente.

Uma das aplicações mais comuns do processo de micronização consiste no reprocessamento da borracha de pneus descartados, isso se deve ao fato de o material não ser passível de reciclagem pelos processos tradicionais, já que se trata de um termofixo (ZHANG et al., 2009). Nesse caso, a conversão do material em pó e posterior mistura com polímero termoplástico resulta na criação de um elastômero termoplástico passível de moldagem por injeção, extrusão e outros processos convencionalmente utilizados na indústria de transformação de polímeros (ZHANG et al., 2009). Diversos estudos (AYYER et al., 2013; ZHANG et al., 2009; COLOM et al., 2007; 
SONNIER et al., 2008) foram conduzidos abordando os aspectos da aplicação de micronização na recuperação de borracha de pneus e posterior processamento com outros polímeros. Os resultados demonstraram que a técnica é viável.

Outras pesquisas (FERNANDES et al., 2008; JUNIOR et al., 2008) estudam a aplicação da micronização em fibras naturais para desenvolvimento de materiais compósitos. De acordo com Junior et al. (2008), nesses casos, o processo de micronização é importante por garantir a homogeneidade da mistura, melhorando as propriedades do material final.

Assim sendo, esse estudo buscou analisar a possibilidade de aplicação da micronização na reciclagem de produtos multi-materias co-injetados. Para tanto, foi conduzido um estudo prático no qual a micronização foi utilizada na reciclagem de escovas de dente multi-materiais co-injetadas.

\section{MATERIAIS E MÉTODO}

\section{Materiais}

Foram utilizados os seguintes materiais:

- Escovas de dente multi-materiais co-injetadas de cinco marcas diferentes. As escovas foram adquiridas em estado pré-consumo em suas embalagens originais.

- Polietileno de baixa densidade linear (PEBDL) adquirido no mercado com índice de fluidez de $10 \mathrm{~g} / 10 \mathrm{~min}$.

\section{Método}

Os procedimentos realizados foram os seguintes:

1. Identificação dos materiais do produto: os materiais presentes nas escovas foram identificados por FTIR. O equipamento utilizado foi um Perkin Elmer ${ }^{\circledR}$, modelo Spectrum 100 com resolução de 4cm-1 realizando 16 varreduras por amostra. O referido equipamento pertence ao Laboratório de Design e Seleção de Materiais (LdSM) da UFRGS. Os espectros gerados no FTIR foram posteriormente comparados a espectros da biblioteca digital da marca Sadtler ${ }^{\circledR}$ com número de série 41285 .

Para identificação do material metálico encontrado no interior das escovas foi utilizado um espectrômetro de fluorescência por Raio-X da marca Bruker ${ }^{\circledR}$, modelo S1 Turbo sd. O referido equipamento pertence ao LdSM/UFRGS.

2. Micronização à temperatura ambiente: devido ao tamanho das escovas, as mesmas foram trituradas antes da micronização. Para tanto, foi utilizado um triturador da marca SEIBT®, modelo MGHS 15/85 com rotação de 1125 rpm e peneira de $8 \mathrm{~mm}$. O equipamento pertence à Universidade Feevale.

A micronização foi realizada em um equipamento da marca $A X$ Plásticos ${ }^{\circledR}$ com rotação de $1.600 \mathrm{rpm}$. O micronizador utilizado pertence à Universidade Feevale. $O$ material foi passado duas vezes no micronizador, na primeira vez foi utilizada uma peneira de $3 \mathrm{~mm}$. Na segunda micronização foi utilizada uma peneira de $0,5 \mathrm{~mm}$. 
3. Identificação do tamanho das partículas geradas: a identificação do tamanho médio das partículas geradas na trituração e nas duas micronizações foi realizado através de medidas realizadas no microscópio eletrônico de varredura (MEV). O equipamento é da marca Hitachi®, modelo TM 3000 com aumento de até 30.000 vezes. O referido microscópio pertence ao LdSM/UFRGS.

As aproximações utilizadas variaram de 40x a 50x nas amostras da trituração e da primeira micronização. Na segunda micronização as aproximações foram de 50x e 100x. Diferentes partículas foram medidas nas amostras, devido à irregularidade das mesmas, cada partícula foi medida em sua maior dimensão (largura ou altura).

4. Extrusão: antes da extrusão os materiais foram secos em estufa a $60^{\circ} \mathrm{C}$ por 24h. Os materiais foram misturados nas concentrações e quantidades informadas na Tabela 1.

\begin{tabular}{|l|l|l|}
\hline & Concentração (\%) & Quantidade (g) \\
\hline PE Virgem & 70 & 583 \\
\hline Escovas micr. & 30 & 175 \\
\hline TOTAL & 100 & 758 \\
\hline
\end{tabular}

Tabela 1- Concentração e quantidade dos materiais utilizados para o estudo de reciclagem Fonte: Elaborado pelos autores

A extrusão foi realizada em um equipamento de rosca simples da marca SEIBT®, modelo MR6D59. O perfil de temperatura utilizada foi (da Zona 1 a Zona 5): $160^{\circ} \mathrm{C}$, $180^{\circ} \mathrm{C}, 180^{\circ} \mathrm{C}, 190^{\circ} \mathrm{C}$ e $200^{\circ} \mathrm{C}$. Sendo a Zona 1 a entrada e a Zona 5 a saída. A velocidade da rosca foi de $35 \mathrm{rpm}$ o que resultou em um tempo de residência das amostras dentro da extrusora de 40 segundos.

Os fios gerados na extrusão foram enrolados e depois pelletizados em um picotador da marca AX Plásticos, modelo AX GRAN. Tanto a extrusora, como o picotador pertencem à Oficina de Modelos e Protótipos da Faculdade de Arquitetura, Urbanismo e Design da UFRGS.

5. Análise da estrutura interna do material extrudado: a análise da estrutura interna do material gerado na extrusão foi realizado por meio de microscopia eletrônica de varredura (MEV). O equipamento é da marca Hitachi®, modelo TM 3000 com aumento de até 30.000 vezes pertencente ao LdSM/UFRGS. As aproximações utilizadas foram 100 e 1.000 vezes.

\section{RESULTADOS E DISCUSSÃO}

Identificação dos materiais do produto: A partir das análises de FTIR realizadas nos materiais das escovas de dente, foi identificado que todos os modelos são constituídos de polietileno (PE), como seu material base (nomeado aqui como material do corpo do produto). Foi identificado ainda que o material que constitui o detalhe dos modelos 1, 2, 4 e 5 é borracha de etileno-polipropileno-dieno (EPDM). A única exce- 
ção, o modelo 3, é constituído de PE tanto no corpo como no detalhe.

O material das cerdas é reconhecido pela literatura como sendo poliamida (PA) (GROVER et al., 2012; ROSEMA et al., 2015). Para confirmar essa afirmação, as cerdas do modelo 4 foram analisadas por FTIR. O resultado confirmou que são constituídas de poliamida (PA). Para uma melhor visualização dos resultados da identificação dos materiais, os mesmos são apresentados na Figura 1. Da mesma maneira, os gráficos comparativos dos espectros de FTIR do material analisado e do padrão encontrado são apresentados nas Figuras de 2 a 4.

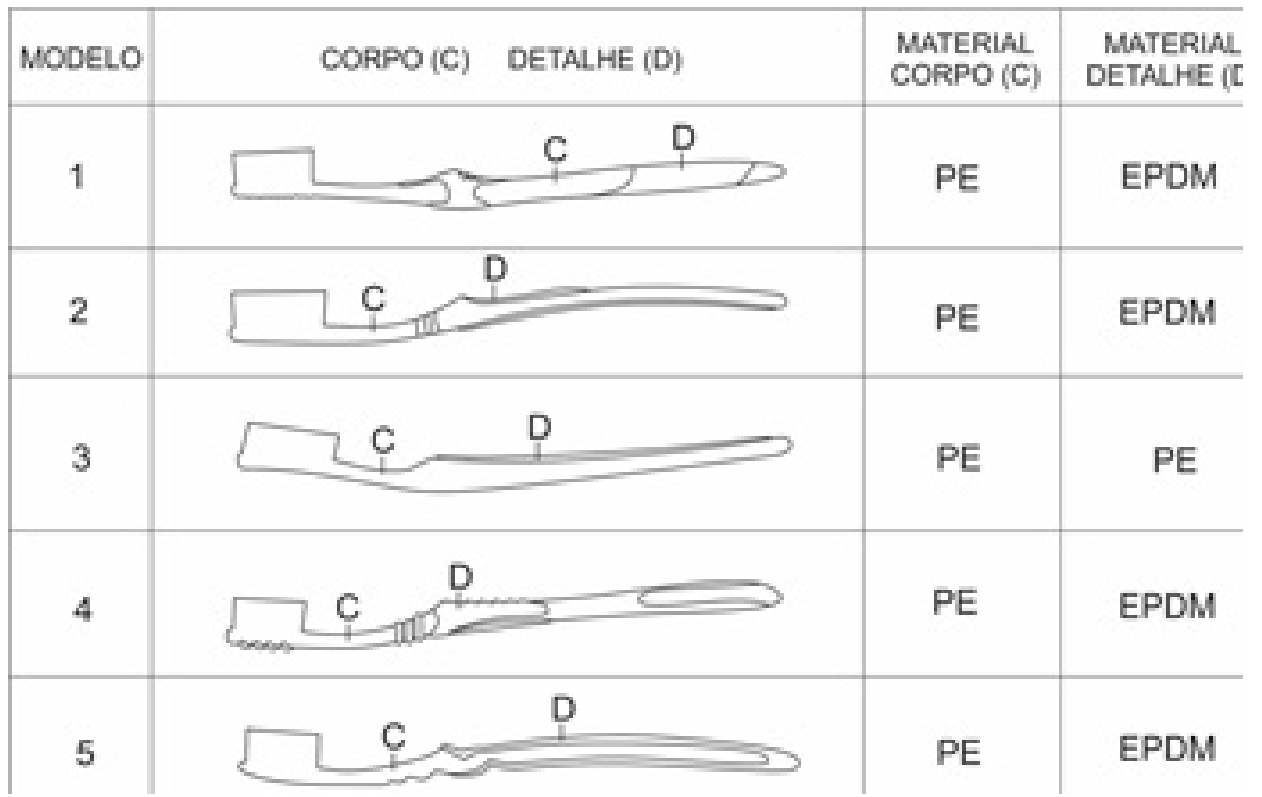

Figura 1- Identificação dos materiais dos produtos utilizados no estudo Fonte: Elaborado pelos autores

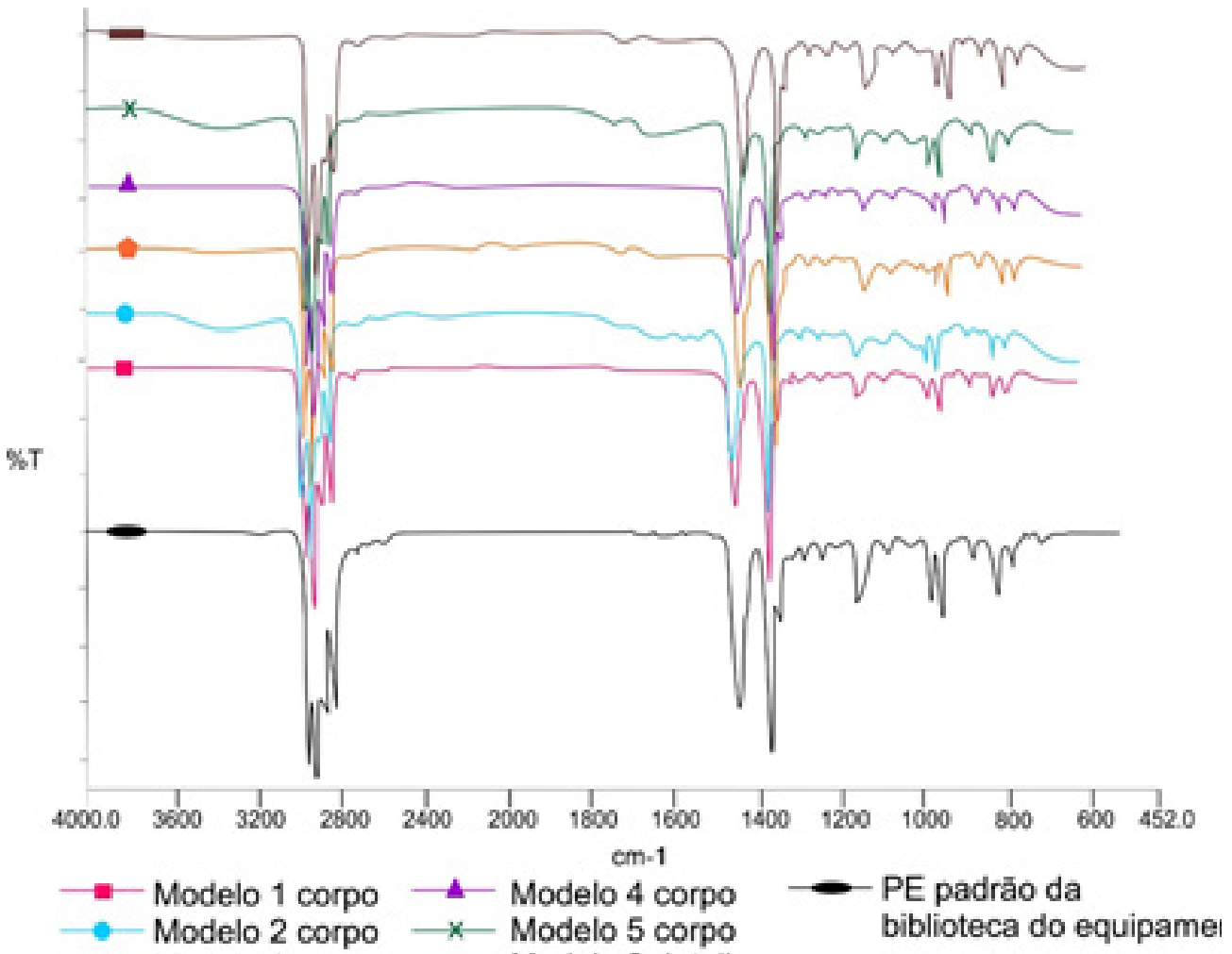

Figura 2 - Espectros de FTIR das escovas de dente caracterizando que o material utilizado na fabricação é Polietileno (PE) Fonte: Elaborado pelos autores 


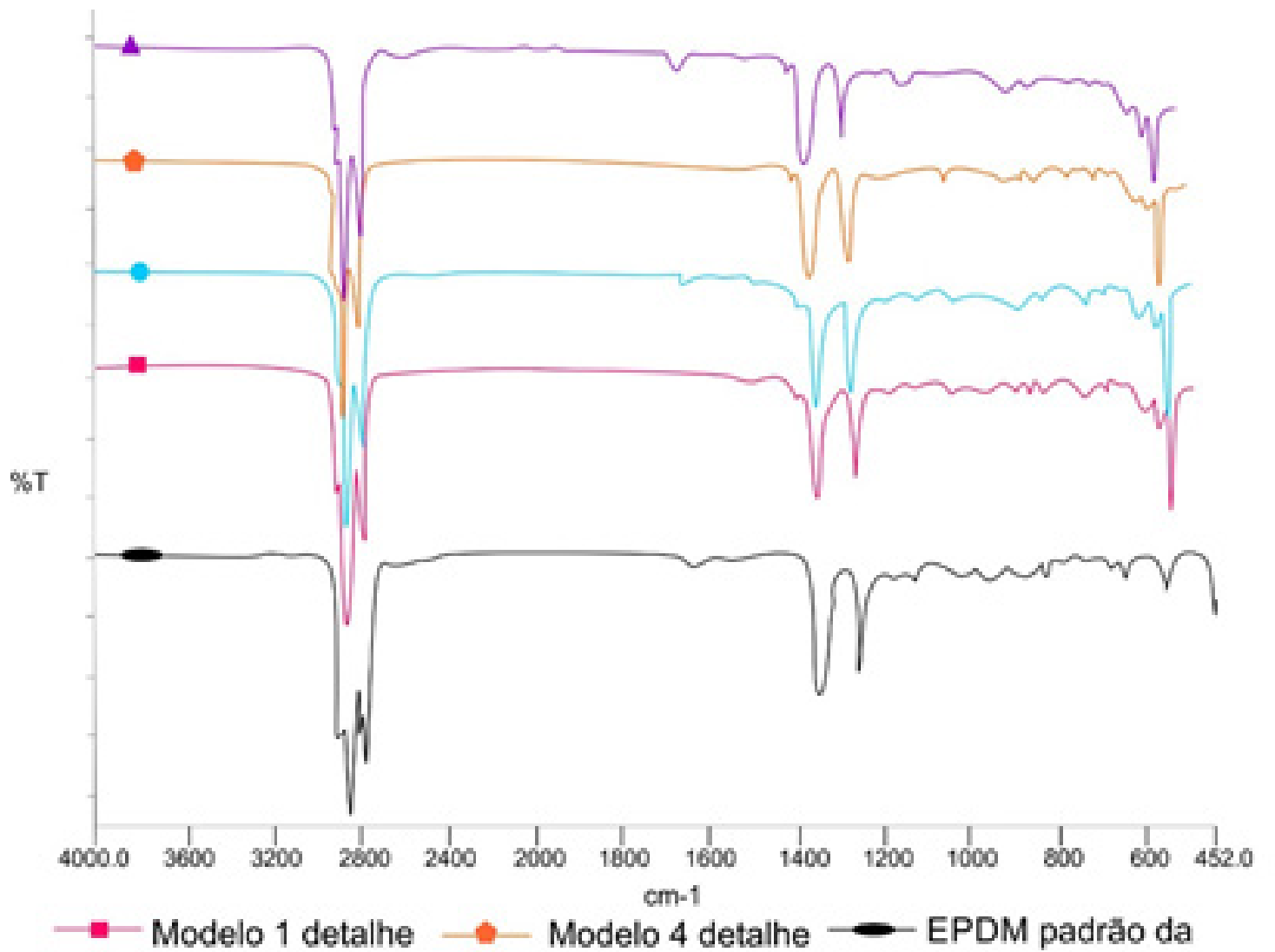

Figura 3 - Espectros de FTIR que caracterizaram a presença de EPDM nos detalhes das escovas de dente Fonte: Elaborado pelos autores

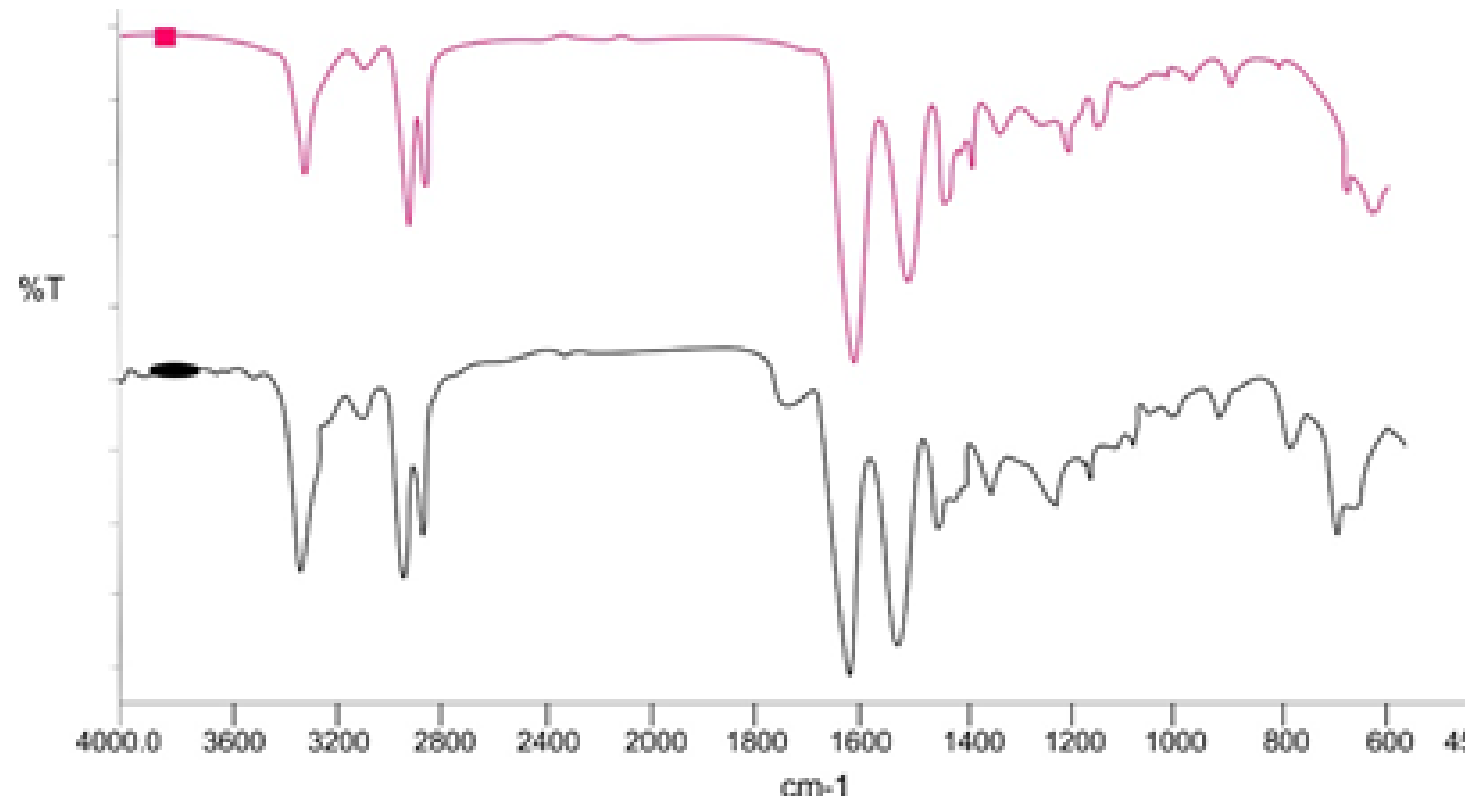

$\longrightarrow$ Modelo 4 cerdas

PA padrăo da biblioter

Figura 4 - Espectro de FTIR que caracterizou Poliamida (PA) como material das cerdas Fonte: Elaborado pelos autores

Durante a extração das cerdas para análise no equipamento, foi constatada a presença de material metálico em todos os modelo de escovas de dente analisados. Estes metais possuem dimensões menores que $5 \mathrm{~mm}$, cuja função é a fixação das cer- 
das no corpo das escova. O referido material metálico foi identificado como uma liga de latão composta basicamente por cobre e zinco.

Micronização a temperatura ambiente: O material se comportou bem na trituração e na micronização. Tanto na trituração como na primeira micronização, as cerdas não foram moídas, passaram pela peneira mantendo o formato original. A concentração de cerdas longas não atrapalhou o processo. Bastou pressionar o material em direção à lâmina para que o mesmo passasse sem entupir o equipamento.

$\mathrm{Na}$ segunda micronização, boa parte das cerdas foram moídas, ganhando a consistência de um pó, apenas alguns fios mais longos foram visíveis ao manusear o material micronizado. Quanto aos pedaços metálicos, ficou difícil de identifica-los visualmente entre o material micronizado, entretanto, alguns foram identificados e, aparentemente, passaram pela peneira com seu tamanho reduzido. Os polímeros mais macios formaram poucas partículas maiores, já o material do corpo, aparentemente, teve seu tamanho consideravelmente reduzido. A Figura 5 representa o material gerado em cada etapa.
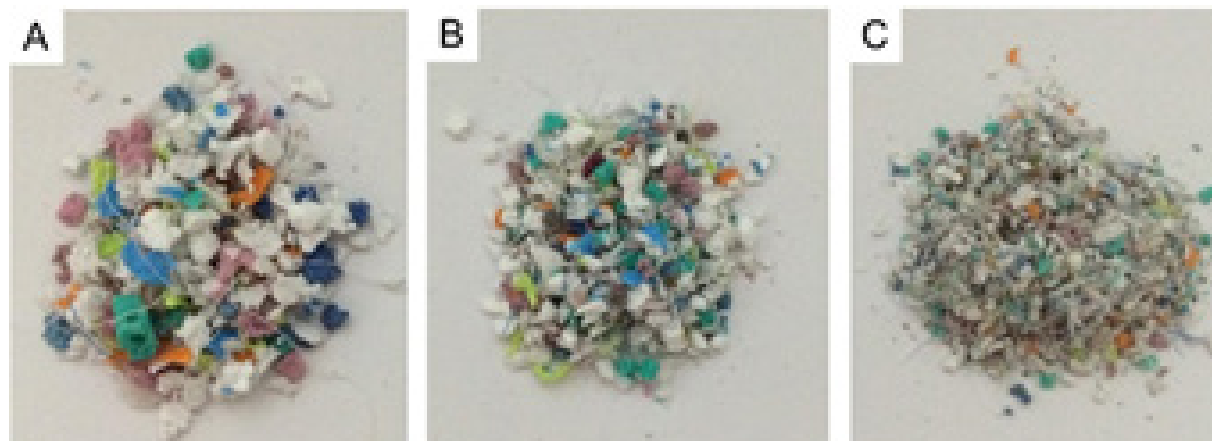

Figura 5 - Comparativo visual do tamanho das partículas geradas nos processos de trituração (A), primeira micronização (B) e segunda micronização $(C)$

Fonte: Elaborado pelos autores

Identificação do tamanho das partículas geradas: As medições realizadas em imagens de microscopia eletrônica de varredura (MEV) proporcionaram um resultado mais exato. Foi constatado que o tamanho médio das partículas geradas na trituração foi de 3,7mm a 3,2mm. As imagens de duas medições são apresentadas na Figura 6.
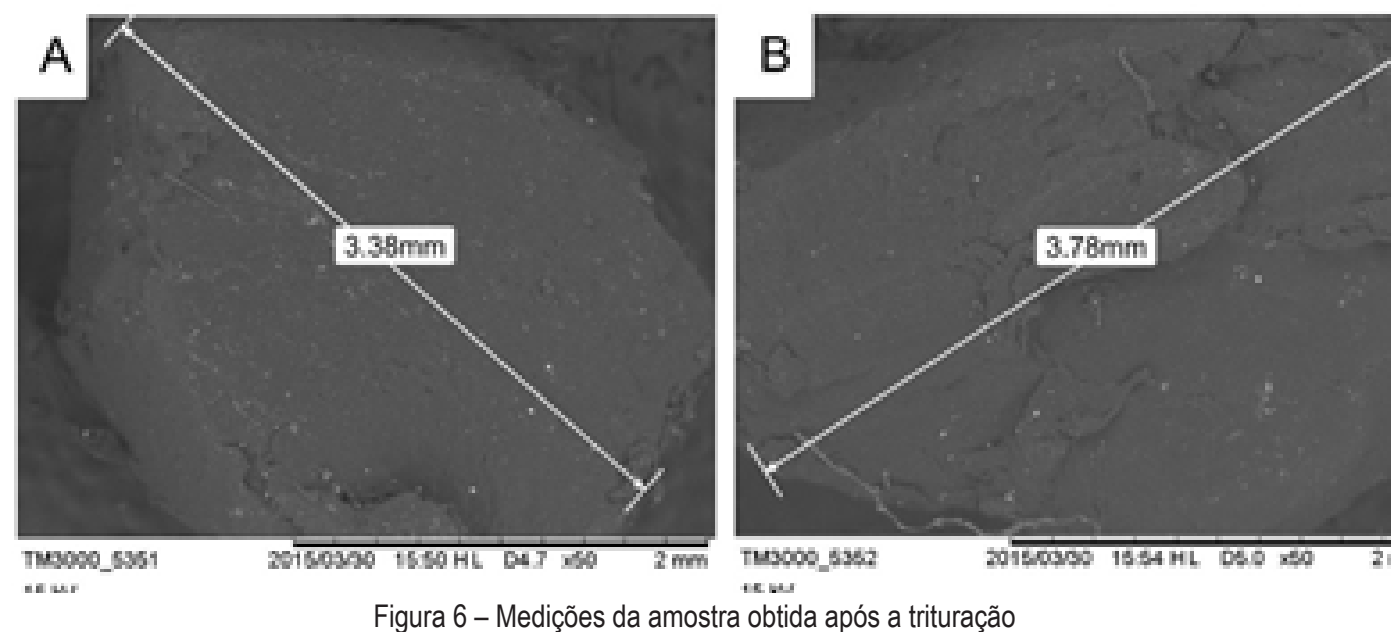

Fonte: Elaborado pelos autores 
Na primeira micronização houve uma maior irregularidade entre o tamanho das partículas. Nesse caso, foi identificado um tamanho que varia entre $3,2 \mathrm{~mm}$ e $1,7 \mathrm{~mm}$ (Figura 7).
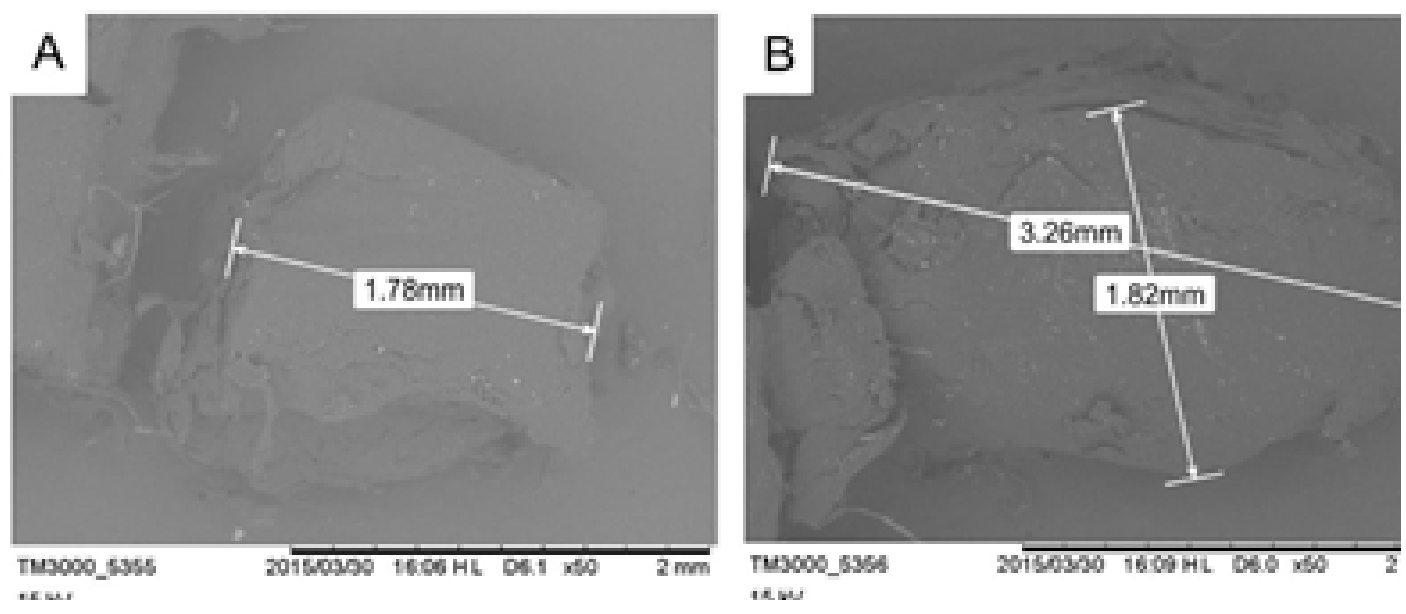

Figura 7 - Medições da amostra obtida após a primeira micronização Fonte: Elaborado pelos autores

Por fim, a medição da amostra gerada após a segunda micronização (Figura 8) revelou grande irregularidade entre o tamanho das partículas. Se observou uma grande quantidade de partículas muito finas (entre $0,3 \mathrm{~mm}$ e $0,08 \mathrm{~mm}$ ) entre algumas partículas maiores $(1,4 \mathrm{~mm}$ e o,6 $\mathrm{mm})$.

A Tabela 2 apresenta a variação média do tamanho das partículas geradas na trituração e nas duas micronizações.
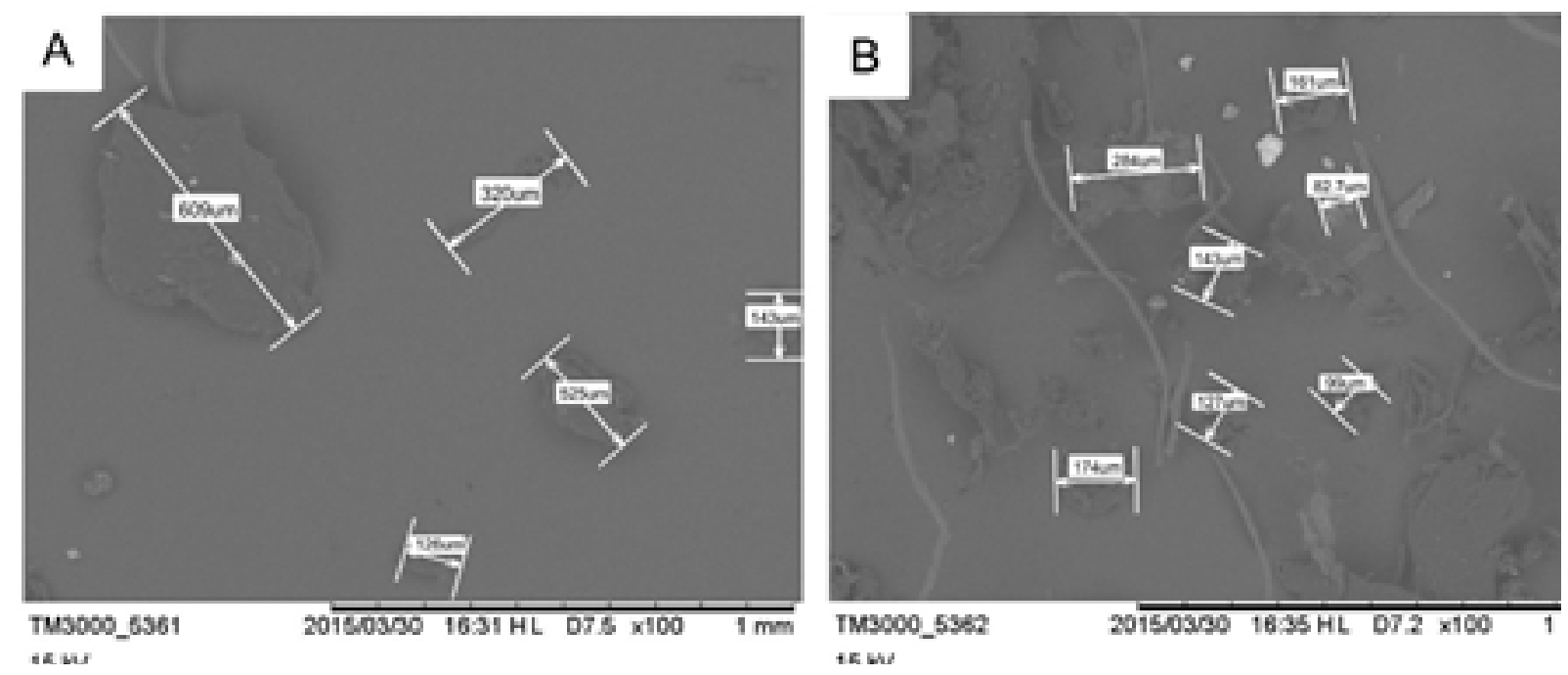

Figura 8- Medições da amostra obtida após a segunda micronização

Fonte: Elaborado pelos autores 


\begin{tabular}{|l|l|}
\hline & Variação do tamanho médio \\
\hline Trituração & $3,7 \mathrm{~mm}$ a $3,2 \mathrm{~mm}$ \\
\hline $1^{\text {a }}$ Micronização & $3,2 \mathrm{~mm}$ a $1,7 \mathrm{~mm}$ \\
\hline $2^{\mathrm{a}}$ Micronização & $1,4 \mathrm{~mm}$ a $0,1 \mathrm{~mm}$ \\
\hline
\end{tabular}

Tabela 2- Variação do tamanho médio das partículas geradas Fonte: Elaborado pelos autores

Extrusão: O material obtido na extrusão teve aparência homogênea, com exceção dos pequenos pedaços metálicos que ficaram aparentes no fio extrudado. Muitos deles se desprenderam do material durante o picotamento. $O$ material não sofreu degradação visível durante o processo.

Análise da estrutura do material extrudado: Através das imagens de MEV (Figuras 9 e 10), foi possível perceber que o material obtido apresentou duas fases, entretanto, os domínios do micronizado estão bem distribuídos na matriz.

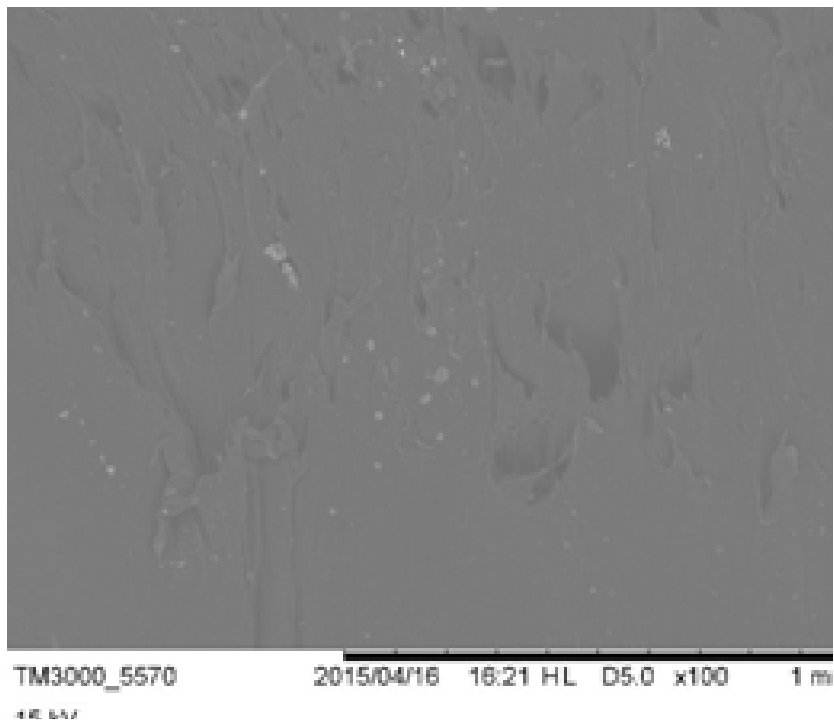

Figura 9 - Imagem de MEV do material extrudado (aproximação de 100x) Fonte: Elaborado pelos autores

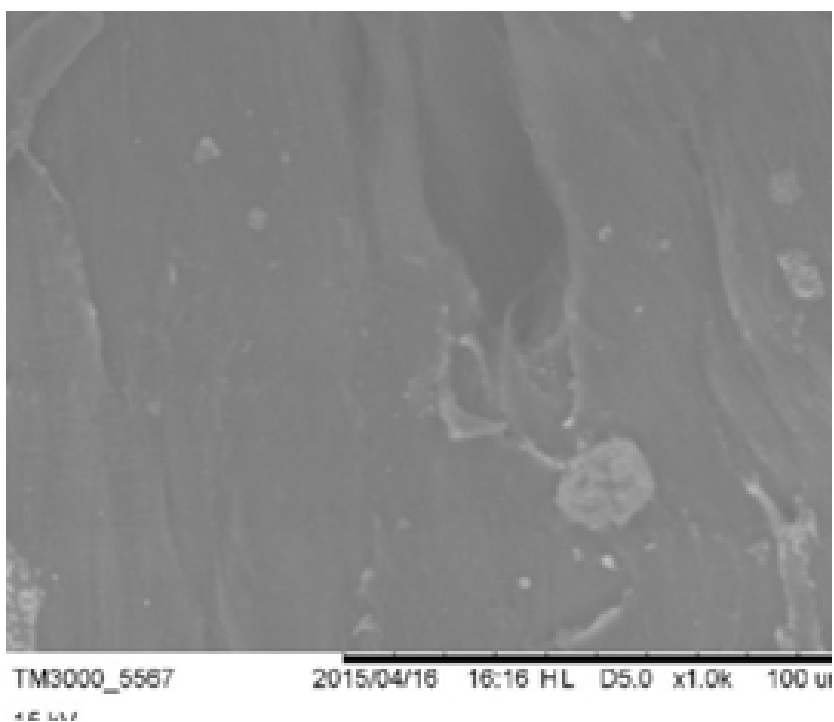

Figura 10 - Imagem de MEV do material extrudado (aproximação de 1.000x) Fonte: Elaborado pelos autores 


\section{CONCLUSÃO}

Diante dos resultados obtidos, é possível concluir que o processo de micronização pode ser aplicado na reciclagem de escovas de dente multi-materiais. O processo analisado mostrou-se factível, com utilização de metodologia usual, simples e acessivel industrialmente. Além disso, não houve degradação do material, pois o mesmo se mostrou estável ao longo do processo, assim como o material não danificou os equipamentos utilizados.

No que diz respeito ao aproveitamento de material, o estudo demonstrou a possibilidade de reciclagem do produto inteiro, o que representa uma baixa geração de rejeito na reciclagem. O único rejeito constatado foi a perda de material mínima e inerente aos processos de micronização e extrusão.

O estudo contribuiu com a geração de alternativas para o reprocessamento de produtos multi-materiais. Tal resultado impacta no desenvolvimento científico e tecnológico, uma vez que o mesmo vai ao encontro dos ideais de sustentabilidade, tema de relevância mundial.

\section{AGRADECIMENTOS}

À CAPES e ao CNPq pelas bolsas de pesquisa e infraestrutura. Lembra-se também da Universidade Feevale pelo apoio e disponibilização dos equipamentos de trituração e micronização. E por fim, ao Instituto de Química/ UFRGS, em especial ao Laboratório de Polímeros Avançados (LPA) pelo apoio.

\section{REFERÊNCIAS}

ADVANI, S; HSIAO, K. Manufacturing Techniques for Polymer Matrix Composites (PMCs), Woodhead Publishing: Philadelphia, 2012.

AGUADO, J.; SERRANO, D. Feedstock Recycling of Plastic Wastes, Royal Society of Chemistry: Cambridge, 1999.

ALLWOOD, J.; CULLEN, J. Sustainable materials with both eyes open, UIT Cambridge: Cambridge, 2012. Disponivel em: www.withbotheyesopen.com Acessado em $11 / 01 / 2015$.

ANTUNES, A. Setores da Indústria Química Orgânica, E-papers: Rio de Janeiro, 2007.

ASHBY, M. Materials Selection in Mechanical Design, Oxford: Elsevier, 2011.

ASHBY, M.; JOHNSON, K. Materials and Design: The art and science of material 
selection in product design, Oxford: Butterworth-Heinemann, 2002.

ASSADOURIAN, E. Transforming Cultures: From Consumerism to Sustainability, Journal of Macromarketing, 30: 186, 2010.

AYYER, R. et al. Effects of Micronized Rubber Powders on Structure and Properties of Polypropylene Composites, Waste Biomass Valor, 4:65-71, 2013.

BOOTHROYD, G. et al. A Product Design for Manufacture and Assembly. 3rd edition, CRC Press: Florida, 2011.

CETELEM. Pesquisa Observador Brasil 2011. Disponível em: http://www.cetelem. com.br/portal/Sobre_Cetelem/Observador.shtml. Acessado em 15/06/2014.

COLOM, X. et al. Composites reinforced with reused tyres: Surface oxidant treatment to improve the interfacial compatibility, Composites: Part A. p. 44 - 50, 2007.

FERNANDES, B. et al. Avaliação da secagem de fibras de coco a $100^{\circ} \mathrm{C}$, visando sua micronização para utilização em compósitos poliméricos, Revista Brasileira de Produtos Agroindustriais. V. 10, no 2, p.141-148, 2008.

FERRANTE, M.; WALTER, Y. A materialização da ideia: noções de materiais para design de produto, Rio de Janeiro: LTC, 2010.

GAZIULUSOY, A. et al. System innovation for sustainability: a systemic double-flow scenario method for companies. Journal of Cleaner Production, v. 45, p. 104-116, 2013.

GROVER, D. et al. Toothbrush 'A key to mechanical plaque control. Indian Journal of Oral Sciences. V. 3, issue 2, p. 62-68, 2012.

HALLSTEDT, S. et al. Key elements for implementing a strategic sustainability perspective in the product innovation process, Journal of cleaner production, v. $51, p$. 277-288, 2013.

HOPEWELL, J. et al. Plastics recycling: challenges and opportunities. Philosophical Transactions of the Royal Society. N. 364, p. 2115-2126, 2009.

ICHINOSE, D.; YAMAMOTO, M. On the relationship between the provisiono $f$ waste management and ilegal dumping, Resource and Energy Economics. N. 33, p. 79-93, 2011.

JIMÉNEZ, E. et al. Environment, Energy and Climate Change I. Springer: Switzerland, 2015.

JUCÁ, J. Disposição final dos resíduos sólidos urbanos no Brasil. 5 Congresso Brasi- 
leiro de Geotecnia Ambiental. Porto Alegre, 2003.

JULIER, G. The culture of design, 3rd edition, SAGE publications Ltda, 2013.

JUNIOR, C. et al. Avaliação do balanço energético de micronização de fibras vegetais para aplicações tecnológicas em novos materiais, Revista Ciências Exatas, Universidade de Taubaté (UNITAU). Vol. 8, nำ1, 2008.

KARANA, E. et al. Materials experience: Fundamentals of materials and design. Oxford: Butterworth-Heinemann, 2014.

KIM, N.; ISAYEV, A. Birefringence and Interface in Sequential Co-Injection Molding of Amorphous polymers: Simulation and Experiment, Polymer Engineering and Science, p. 88-106, 2015.

KOLLIKKATHARA, N. et al. A system dynamic modeling approach for evaluating municipal solid waste generation, landfill capacity and related cost management issues, Waste Management: 30, 2010.

KROMM, F. et al. Definition of a multimaterials design method, Materials and Design, n. 28 p. 2641-2646, 2007.

KOUSHAL, V. et al. Plastics: Issues Challenges and Remediation, Journal of Waste Resources. Vol. 4 nำ1, 2014.

LAGARINHOS, C.; TENÓRIO, J. Tecnologias Utilizadas para a Reutilização, Reciclagem e Valorização Energética de Pneus no Brasil, Polímeros, v.18, n.2, p.106-118, 2008.

MALEQUE, M.; SALIT, M. Materials Selection and Design, Singapore: Springer Singapore, 2013.

MANZINI, E.; VEZZOLI, C. O Desenvolvimento de Produtos Sustentáveis - Os requisitos ambientais dos produtos industriais, Edusp: São Paulo, 2002.

MEYER, R. Packaging: Sustaining Cultures and Meeting new Imperatives. In Handbook of Recycling: State-of-the-art for practioners, analysts and scientist. WORRELL, E.; REUTER, M. (editors). Elsevier, 2014.

MORSELLI, L. et al. Waste recovery: Strategies, techniques and applications in Europe, FrancoAngeli: Milão, 2009.

NKWACHUKWU, O. et al. Focus on potential environmental issues on plastic world towards a sustainable plastic recycling in developing countries, International Journal of Industrial Chemistry, 4:34, 2013.

ORGANIZAÇÃO DAS NAÇÕES UNIDAS - ONU. UN News Centre: World population 
projected to reach 9.6 bilion by 2050 - UN report, 2013. Disponível em: http:// www.un.org/apps/news/story.asp?NewsID=45165\#.U6tt8fldUww Acessado em 13/06/2013.

PLATCHEK, E. Design industrial: metodologia para o desenvolvimento de produtos sustentáveis, São Paulo: Atlas, 2012.

PINTO, J. et al. Impactos ambientais causados pelos plásticos - Uma discussão abrangente sobre os mitos e dados científicos, segunda edição. E-papers: Rio de Janeiro, 2012.

RAJENDRAN, S. et al. Environmental impact assessment of composites containing recycled plastics, Resources, Conservation and Recycling, 60 pp. 131 - 139, 2012.

ReVELLE, J. (editor). Manufacturing Book of Best Pactices: An innovation, productivity and quality focus, CRC Press: Florida, 2002.

ROSEMA, N. A. M. et al. The efficacy of powered tothbrushes following a brushing exercise: a systematic review. International Journal of Dental Hygiene. DOI: 10.1111/ idh.1, 2015.

SIMÕES, C. et al. Environmental and economic assessment of Road safety product made with virgin and recycled HDPE: A comparative study, Journal of Environmental Management, v. 114, p. 209-215, 2013.

SONNIER, R. et al. Compatibilizing thermoplastic/ground tyre rubber powder blends: Efficiency and limits, Polymer Testing. № 27, p. 901 - 907, 2008.

THOMAS, S.; YANG, W. Advances in Polymer Processing: from macro to nano scales, Woodhead Publishing: Cambridge, 2009.

VAN BEZOOYEN, A. Materials Driven Design. Chapter 19 in KARANA, E. et al. Materials experience: Fundamentals of materials and design. Oxford: Butterworth-Heinemann, 2014.

WARGNIER, W.; et al. Proposal for a multi-material design procedure, Materials and Design, n. 56, p. 44-49, 2014.

WILSON, D. et al. Role of informal sector recycling in waste management in developing countries, Habitat International. V. 30, Issue 4, p. 797-808, 2006.

WIMMER, W. et al. Ecodesign: The competitive advantage, Springer:London, 2010.

WORRELL, E.; REUTER, M. (editors). Handbook of Recycling: State-of-the-art for practioners, analysts and scientist, Elsevier: Oxford, 2014. 
ZAVERL, M.; et al. Study of the Effect of Processing Conditions on the Co-Injection of PBS/PBAT and PTT/PBT Blends for Parts with Increased Bio-Content, Journal of applied polymer science, DOI 10.1002, 2013.

ZHANG, S. et al. Characterization of the properties of thermoplastic elastomers containing waste rubber tire poder, Waste Management, № 29, p. 1480 - 1485, 2009. 